\title{
65789
}

\section{NUTATIONAL STABILITY OF A DUAL-SPIN SATELLITE UNDER THE INFLUENCE OF APPLIED REACTION TORQUES}

\section{PETER M. BAINUM}

\section{SEPTEMBER 1971}

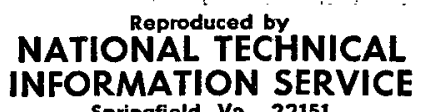

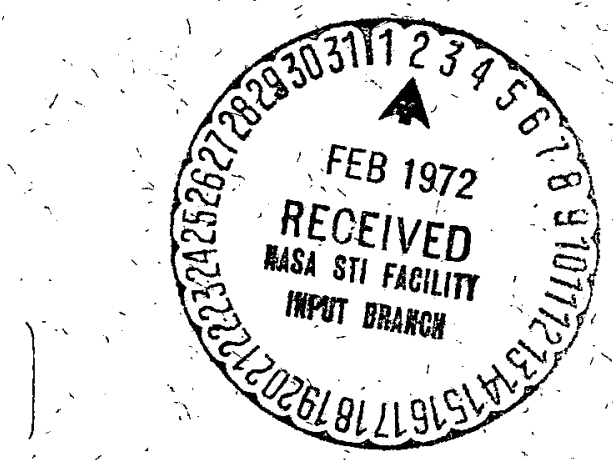

CDEENRFIT MARYLAND

(NASA-TH-X-65789) NUTACIONAL STABILITY OF
A DUAL-SPIN SATELLITE UNDER THE INFLUENCE
OF APELIED REACTIOA TORQUES P. B. BAIRUA
(NASA) SEP. $197133 \mathrm{p}$ $\ldots-$
N72-15597

Unclas 13619 


\section{NUTATIONAL STABILITY OF A DUAL-SPIN SATELLITE UNDER THE INFLUENCE OF APPLIED REACTION TORQUES}

Peter M. Bainum

September 1971

GODDARD SPACE FLIGHT CENTER

Greenbelt, Maryland 


\title{
NUTATIONAL STABILITY OF A DUAL-SPIN SATELLITE \\ UNDER THE INFLUENCE OF \\ APPLIED REACTION TORQUES
}

Peter M. Bainum

\begin{abstract}
The nutational stability of a dual-spin satellite under the influence of an applied reaction torque is analyzed. It is assumed that since the solar paddle attachments to the hub of the spacecraft are not rigidly locked, the effect of the solar panels can be replaced by a constant reaction torque acting on the hub of the spacecraft. This could result in the satellite having an equilibrium motion about an axis displaced from the nominal axis of symmetry. The variational equations of motion are developed about such an equilibrium position using the SAS-A spacecraft as a model. Energy dissipation on the rotor as well as the main body is included. This nonautonomous set of differential equations are linearized and transformed to an autonomous set using the Lyapunov Reducibility Theorem. The stability of the kinematically similar system is examined numerically using representative SAS-A parameters for the case when either pair of solar panels is assumed to be loosely attached. Stability is verified for small system nutation angles (0.1 degree) although the time constant associated with the least damped mode is approximately one order of magnitude larger than that for the SAS-A system without the influence of the reaction torques.
\end{abstract}


$\mathrm{A}, \mathrm{B}, \mathrm{C}=$ main body moments of inertia about the $\mathrm{x}, \mathrm{y}, \mathrm{z}$ axes respect $\overline{\mathrm{A}}, \overline{\mathrm{B}}, \overline{\mathrm{C}}=$ composite moments of inertia about the $\mathrm{x}, \mathrm{y}, \mathrm{z}$ axes respect $\mathrm{A}(\mathrm{t}) \quad=$ system matrix, appears when linear equations are written $\mathrm{i}$ vector form

$b_{i}=$ unit vectors along the $x, y, z$ axes respectively $(i=1,2,3)$

$\bar{b}_{i}{ }^{\prime} \quad=\quad$ unit vectors fixed to the nominal plane of the undeflected wh rotating with it

$\mathrm{C}_{\mathrm{i}}=$ coefficients occurring in the linear equations

D = system matrix appearing in the kinematically similar syste equations

$\mathrm{H}=$ magnitude of system angular momentum vector, $\overline{\mathrm{H}}$

$I_{b_{i}}=$ moment of inertia of main body about the $\bar{b}_{i}$ axis

$I_{R_{i}}=$ moment of inertia of rutor about its $\bar{b}_{i}$ axis

$I_{d_{i}}=$ moment of inertia of the pendulous damper about the $\bar{b}_{i}$ axi

$\mathrm{K}=$ the restoring spring constant of the damper torsion wire su

$\mathrm{K}_{\mathrm{R}}=$ the restoring spring constant of the rotor

$\mathrm{k}=$ the nutation damper damping (rate) constant

$\mathrm{k}_{\mathrm{R}} \quad=$ rotor damping constant

$L_{d_{i}}=$ the applied reaction torque about the $\bar{b}_{i}$ axis 


\begin{tabular}{|c|c|c|}
\hline$\ell$ & $=$ & height of damper plane above $x, z$ plane \\
\hline M & $=$ & the mass of the main satellite and the rotor \\
\hline$\overline{\mathrm{M}}$ & $=$ & the total system mass \\
\hline $\mathrm{m}$ & $=$ & the pendulum end mass \\
\hline $\mathrm{m}_{\mathrm{R}}$ & $=$ & the mass of the rotor \\
\hline $\mathbf{r}$ & $=$ & radial coordinate of a differential mass on the rotor \\
\hline$r_{0}$ & $=$ & $\begin{array}{l}\text { the distance from the nominal spin }(y) \text { axis to the pendulum hinge } \\
\text { point }\end{array}$ \\
\hline $\mathbf{r}_{1}$ & $=$ & the length of the pendulum \\
\hline $\mathrm{S}(\mathrm{t})$ & $=$ & $\begin{array}{l}\text { bounded nonsingular transformation matrix used to transform } \\
\text { nonautonomous system of equations to an autonomous set }\end{array}$ \\
\hline $\mathbf{s}$ & $=$ & spin rate of rotor relative to main body \\
\hline t & $=$ & time \\
\hline $\mathrm{x}, \mathrm{y}, \mathrm{z}$ & $=$ & principal axes of main satellite \\
\hline $\mathrm{y}_{\mathrm{R}}$ & $=$ & $\begin{array}{l}\text { displacement coordinate of } \mathrm{dm}_{R} \text { from plane of undeflected rotor } \\
\text { disc }\end{array}$ \\
\hline
\end{tabular}


a $=$ angle between the main body symmetry axis $\left(\bar{b}_{2}\right)$ and the main body angular velocity vector

$a_{z}, a_{x}=$ rotor deflection angles about the $\bar{b}_{3}, \bar{b}_{1}$, axes respectively

$\beta_{1}, \beta_{2}=$ transformed rotor deflection coordinates in the kinematically similar system

$\delta, \epsilon, \psi=$ variational coordinates corresponding to perturbations in $\omega_{2}, \omega_{1}$, and $\omega_{3}$, respectively, from the equilibrium motion

$\Gamma \quad=\ell \mathrm{m} / \overline{\mathrm{M}}$

$\gamma=$ the nutation angle, i.e. the angle between the $\bar{b}_{2}$ axis and $\overline{\mathrm{H}}$

$\Omega \quad=$ nominal main body spin rate

$\omega_{i} \quad=$ angular velocities about the $x, y, z$ axes respectively $(i=1,2,3)$

$\phi \quad=$ position angle of the projection of $\mathrm{dm}_{\mathrm{R}}$ on the plane of the undeflected rotor disc

$\phi_{1}=$ nutation damper displacement angle 


\section{Superscript}

()$^{\prime}$ refers to component in $\bar{b}_{i}{ }^{\prime}$ axes system

( ) indicates differentiation with respect to time

Subs cript

$b_{i}, i \quad$ refers to particular main body axes $(i=1,2,3)$

0 refers to equilibrium value

ss refers to steady state 


\section{ACKNOWLEDGMENT}

The research reported here was conducted at the NASA Goddard Space Flight Center, Stabilization and Control Branch where the author was participating as a Faculty Fellow in the NASA-ASEE 1971 Summer Faculty Fellowship Program. My research colleague was Dr. Joseph V. Fedor of the Stabilization and Control Branch whose continued interest is gratefully acknowledged. In addition, the author would like to express his appreciation to Dr. Arun K. Guha, Postdoctoral Research Associate in the Stabilization and Control Branch for the many illuminating discussions. Thanks are also extended to Mr. Peter Fuechsel of The Johns Hopkins University, Applied Physics Laboratory for calculating and supplying the various moments of inertia for the SAS-A system, and to Messrs. Leonard Moriarity and Jim Andary of the Stabilization and Control Branch for the computer programming. 


\section{NUTATIONAL STABILITY OF A DUAL-SPIN SATELLITE UNDER THE INFLUENCE OF APPLIED REACTION TORQUES}

\section{INTRODUCTION}

NASA Goddard Space Flight Center is currently directing the design and development of a series of Small Astronomy Satellites (SAS) whose purpose is to support orbitally based experiments in modern astronomy. The first of these satellites, SAS-A, is a dual-spin spacecraft which has the capability of scanning the entire celestial sphere to determine the relative position and intensity of $\mathrm{X}$-ray emitting sources with respect to the fixed position of the stars. It is important that the attitude of the satellite be precisely known and maintained in order to accurately determine the location of the X-ray emitting sources. The attitude control system for the SAS-A satellite has been designed and developed by the Applied Physics Laboratory of the Johns Hopkins University.

An analysis of the attitude motion and stability of such a dual-spin spacecraft with damping only on the slowly spinning main part was reported previously. ${ }^{1}$ The resulting differential equations of rotational motion when linearized were an autonomous set of fifth order equations with constant coefficients. Analytical stability criteria were developed from these equations using the method of Routh-Hurwitz.

Subsequent to this analysis, it was demonstrated by static and dynamic tests of the SAS-A momentum wheel that there was some energy dissipation in the shaft-momentum wheel assembly. A recent investigation ${ }^{2}$ incorporated the effects of momentum wheel damping into the rotational equations of motion for the SAS-A spacecraft, and analytically considered the attitude stability of such a 
system. Wheel energy dissipation was modeled by assuming the wheel could flex with two degrees of freedom relative to the hub. The nonlinear attitude equations derived for small wheel flexural motion were a ninth order nonautonomous set. An averaging process was used to determine the zeroth and first order secular perturbations on the behavior of the system nutation angle. From this a general analytic stability criterion was established. A numerical evaluation of this criterion using parameters and measured wheel damping data for the Small Astronomy-A satellite indicated that stability about a zero degree nutation angle is insured by a factor of 128 under normal operating conditions. Numerical integration of the nonlinear equations confirmed the analytic results for special cases.

The SAS-A satellite was launched in December 1970. After initial despin maneuvers and a command to pulse the nutation damper magnet to a more optimum damping constant, the nutation angle amplitude was reduced from about 1 degree to approximately $0.10-0.15$ degree. Although this is within the mission requirement of 0.20 degree, it is about an order of magnitude greater than that predicted by prelaunch computer simulations. ${ }^{3}$ Several hypotheses which might explain this discrepancy are currently under investigation. Among these are: possible periodic fluctuations in solar panel lengths due to thermal expansion/ contraction as the satellite rotates in the sunlight and which could excite nutations; excessive mass unbalance of the satellite in-orbit configuration, in which the state of zero nutation angle may not correspond to the lowest energy state, and where nutation damping may approach a steady-state condition of minimum energy but with non-zero nutation; and thirdly, since the solar paddle attachments to the hub of the spacecraft are not rigidly locked, but rather spring-connected, 
the effect of the (loose) solar paddles could be replaced by a constant reaction torque acting on the spacecraft hub. This could cause the satellite to reach an equilibrium motion about an axis slightly displaced from the nominal figure or symmetry axis, thus possibly explaining the anomalous nutational behavior of the SAS-A system.

This third theory will be the basis for the investigation considered herein. Although the results will be applied directly to the SAS-A spacecraft, it should also be of interest to the designers of other dual-spin systems having nonrigidly connected solar paddles.

\section{ANALYSIS}

It is a well-known textbook problem that a rotating rectangular plate with a constant angular velocity can be forced to rotate about a nonprincipal axis of inertia under the influence of a reaction torque which acts about an axis perpendicular to the plate and rotates with it. ${ }^{4}$ In 1963 Garber studied the stability of a gravity-gradient stabilized satellite under the influence of a constant disturbing (reaction) torque. ${ }^{5}$ He concluded that if this torque were sufficient to produce a non-zero steady-state pitch (in-plane) motion, it could lead to instabilities in the roll-yaw out-of-plane motion. ${ }^{5}$

This idea may be applied to the motion of a spinning satellite with loosely attached solar paddles; a plausible model is shown in Figure 1. A possible equilibrium configuration is described by two body-axis components of the spacecraft spin vector that are non-zero: 


$$
\begin{aligned}
& \omega_{2}=\Omega \cos \alpha \\
& \omega_{1}=0 \\
& \omega_{3}=\Omega \sin \alpha
\end{aligned}
$$

where $a$ is the angle between the axis of rotation and the desired system spin axis, and $\Omega$ is the nominal velue of the satellite spin rate.

The equations of motion for an undamped dual-spin satellite system where the desired axis of rotation is the " $\mathrm{y}$ " axis can be represented by:

$$
\left.\begin{array}{l}
\overline{\mathrm{B}} \dot{\omega}_{2}+\omega_{1} \omega_{3}(\overline{\mathrm{A}}-\overline{\mathrm{C}})=\mathrm{L}_{\mathrm{d}_{2}} \\
\overline{\mathrm{A}} \dot{\omega}_{1}+\omega_{2} \omega_{3}(\overline{\mathrm{C}}-\overline{\mathrm{B}})-\omega_{3} \mathrm{I}_{\mathrm{R}_{2}} \mathrm{~s}=\mathrm{L}_{\mathrm{d}_{1}} \\
\overline{\mathrm{C}} \dot{\omega}_{3}+\omega_{1} \omega_{2}(\overline{\mathrm{B}}-\overline{\mathrm{A}})+\omega_{1} \mathrm{I}_{\mathrm{R}_{2}} \mathrm{~s}=\mathrm{L}_{\mathrm{d}_{3}}
\end{array}\right\}
$$

If we calculate the disturbance torque, $\overline{\mathrm{L}}_{\mathrm{d}}$, necessary to give this system a forced rotation represented by Equation (1), we find that:

$$
\left.\begin{array}{l}
\mathrm{L}_{\mathrm{d}_{2}}=\mathrm{L}_{\mathrm{d}_{3}}=0 \\
\mathrm{~L}_{\mathrm{d}_{1}}=\Omega \sin a\left[\Omega \cos a(\overline{\mathrm{C}}-\overline{\mathrm{B}})-\mathrm{I}_{\mathrm{R}_{2}} \mathrm{~s}\right]
\end{array}\right\}
$$

The variational equations of motion about the equilibrium motion, Equation (1), can be developed by assuming:

$$
\begin{aligned}
& \omega_{2}=\Omega \cos a+\delta \\
& \omega_{1}=\epsilon \\
& \omega_{3}=\Omega \sin a+\psi
\end{aligned}
$$


and substituting, Equation (4) back into the equations of motion (2). After linearization, the equations are of the form:

$$
\left.\begin{array}{l}
\dot{\delta}+\mathrm{C}_{1} \epsilon=0 \\
\dot{\epsilon}+\mathrm{C}_{2} \psi+\mathrm{C}_{3} \delta=0 \\
\dot{\psi}+\mathrm{C}_{4} \epsilon=0
\end{array}\right\}
$$

where

$$
\begin{aligned}
& \mathrm{C}_{1}=(\overline{\mathrm{A}}-\overline{\mathrm{C}}) \Omega \sin \alpha / \overline{\mathrm{B}} \\
& \mathrm{C}_{2}=-\left[(\overline{\mathrm{B}}-\overline{\mathrm{C}}) \Omega \cos \alpha+\mathrm{I}_{\mathrm{R}_{2}} \mathrm{~s}\right] / \overline{\mathrm{A}} \\
& \mathrm{C}_{3}=-[(\overline{\mathrm{B}}-\overline{\mathrm{C}}) \Omega \sin \alpha] / \overline{\mathrm{A}} \\
& \mathrm{C}_{4}=\left[(\overline{\mathrm{B}}-\overline{\mathrm{A}}) \Omega \cos \alpha+\mathrm{I}_{\mathrm{R}_{2}} \mathrm{~s}\right] / \overline{\mathrm{C}}
\end{aligned}
$$

If the first of Equations (5) is multiplied by $C_{4}$, the third by $-C_{1}$, and the result added, the linear dependence between $\delta$ and $\psi$ can be established.

$$
\mathrm{C}_{4} \dot{\delta}-\mathrm{C}_{1} \dot{\psi}=0
$$

or

$$
\mathrm{C}_{4} \delta=\mathrm{C}_{1} \psi+\mathrm{C}_{4} \delta(0)-\mathrm{C}_{1} \psi(0)
$$

where $\delta(0), \psi(0)$ represent the initial conditions in the variational coordinates. After elimination of $\delta$ as a dependent coordinate, the independent variational equations of motion can be written:

$$
\left.\begin{array}{l}
\dot{\epsilon}+\left(\frac{\mathrm{C}_{2} \mathrm{C}_{4}+\mathrm{C}_{3} \mathrm{C}_{1}}{\mathrm{C}_{4}}\right) \psi=-\frac{\mathrm{C}_{3}}{\mathrm{C}_{4}}\left[\mathrm{C}_{4} \delta(0)-\mathrm{C}_{1} \psi(0)\right] \\
\dot{\psi}+\mathrm{C}_{4} \epsilon=0
\end{array}\right\}
$$


The homogeneous system has the following characteristic equation in the variable, $\lambda$,

$$
\lambda^{2}-\left(\mathrm{C}_{2} \mathrm{C}_{4}+\mathrm{C}_{1} \mathrm{C}_{3}\right)=0
$$

For a boundedness of the motion the roots of Equation (9) must be purely imaginary, or

$$
\mathrm{C}_{2} \mathrm{C}_{4}+\mathrm{C}_{1} \mathrm{C}_{3}<0
$$

Inequality (10) may be expanded to take the form:

$$
\begin{aligned}
\overline{\mathrm{B}}[(\overline{\mathrm{A}}-\overline{\mathrm{C}}) \Omega \sin a] & {[(\overline{\mathrm{B}}-\overline{\mathrm{C}}) \Omega \sin a] } \\
+\overline{\mathrm{C}}\left[(\overline{\mathrm{B}}-\overline{\mathrm{C}}) \Omega \cos a+\mathrm{I}_{\mathrm{R}_{2}} \mathrm{~s}\right]\left[(\overline{\mathrm{B}}-\overline{\mathrm{A}}) \Omega \cos a+\mathrm{I}_{\mathrm{R}_{2}} \mathrm{~s}\right]>0 & (11)
\end{aligned}
$$

Inequality (11) is obviously satisfied for the case of a SAS-type spacecraft where $\mathrm{s} \gg \gg>$ and approximately 90 percent of the system momentum is contributed by the rotor.

For non-zero initial conditions in $\delta$ or $\psi$ it is seen from Equation (8) that non-zero steady-state values of $\delta$ and $\psi$ would result, but also that $\epsilon_{\mathrm{ss}}=0$. It is clear that, even in the presence of damping, this system would never be asymptotically stable about the assumed equilibrium motion of Equation (1). Or, in other words, for small initial perturbations in the variational coordinates, regardless of the time elapsed, the system motion would never return exactly to its original equilibrium state.

Up to this point the analysis has considered the forced rotation of a general asymmetric dual-spin satellite without energy dissipation on either the main part or the rotor. To consider the motion and stability of this system with 
energy dissipation, first on the main part, and later on both parts of the spacecraft, a specific model for the energy dissipation must be selected. For this purpose the nutation damping system employed in the SAS-type satellites will be considered.

The satellite, wheel, and damping systems are illustrated in Figure 2. The elements of the attitude control system are: (1) the main part of the spacecraft, essentially a right circular cylinder where the nominal spin axis is the $\bar{b}_{2}$ body axis, (2) a small momentum wheel or rotor assumed to be connected to the primary part near its center of mass, and whose spin axis is nominally parallel to the $\bar{b}_{2}$ body axis, and (3) a pendulous-type nutation damper which is attached to the main part and constrained to move in a plane a distance $\ell$ above the $\bar{b}_{1}, \bar{b}_{3}$ plane (determined by the body axes perpendicular to the symmetry axis, $\bar{b}_{2}$ ). The damper is hinged about a torsion wire support which offers a restoring torque in addition to the dissipative torque associated with the damper motion.

The equations of motion for this system under the reaction torque described above and with energy dissipation on the main body may be expressed:

$$
\left.\begin{array}{l}
\overline{\mathrm{B}} \dot{\omega}_{2}+\omega_{1} \omega_{3}(\overline{\mathrm{A}}-\overline{\mathrm{C}})=\mathrm{L}_{\mathrm{f}_{2}} \\
\overline{\mathbf{A}} \dot{\omega}_{1}+\omega_{2} \omega_{3}(\overline{\mathrm{C}}-\overline{\mathrm{B}})-\omega_{3} \mathrm{I}_{\mathrm{R}_{2}} \mathrm{~s}=\mathrm{L}_{\mathrm{d}_{1}}+\mathrm{L}_{\mathrm{f}_{1}} \\
\overline{\mathbf{C}} \dot{\omega}_{3}+\omega_{1} \omega_{2}(\overline{\mathrm{B}}-\overline{\mathrm{A}})+\omega_{1} \mathrm{I}_{\mathrm{R}_{2}} \mathrm{~s}=\mathrm{L}_{\mathrm{f}_{3}}
\end{array}\right\}
$$

in addition to an equation describing the single-degree-of-freedom damper. The damping terms, $L_{f_{i}}$, and the damper equation as previously derived ${ }^{1}$, can be expanded to yield the following system of first order nonlinear differential equations under the assumptions that: 


$$
\left.\begin{array}{c}
\omega_{\mathrm{i}} / \omega_{2} \ll 1, \dot{\phi}_{1} \ll 1, \phi_{1} \ll 1, \\
\overline{\mathrm{B}} \dot{\omega}_{2}+\omega_{1} \omega_{3}(\overline{\mathrm{A}}-\overline{\mathrm{C}})+\mathrm{mr} \mathrm{r}_{1}\left(\mathrm{r}_{1}+\mathrm{r}_{0}\right) \ddot{\phi}_{1}=0 \\
\overline{\mathrm{A}} \dot{\omega}_{1}+\omega_{2} \omega_{3}(\overline{\mathrm{C}}-\overline{\mathrm{B}})-\omega_{3} \mathrm{I}_{\mathrm{R}_{2}} \mathrm{~s}-2 \mathrm{~m} \omega_{2} \mathrm{r}_{1} \Gamma \dot{\phi}_{1}=\mathrm{L}_{\mathrm{d}_{1}} \\
\overline{\mathrm{C}} \dot{\omega}_{3}+\omega_{1} \omega_{2}(\overline{\mathrm{B}}-\overline{\mathrm{A}})+\omega_{1} \mathrm{I}_{\mathrm{R}_{2}} \mathrm{~s}-\mathrm{m} \mathrm{r}_{1} \Gamma \ddot{\phi}_{1}+\mathrm{m} \omega_{2}^{2} \mathrm{r}_{1} \Gamma \phi_{1}=0 \\
\mathrm{mr}{ }_{1}^{2} \ddot{\phi}_{1}-\mathrm{mr} \mathrm{r}_{1} \Gamma \dot{\omega}_{3}+\mathrm{mr} \mathrm{r}_{1}\left(\mathrm{r}_{0}+\mathrm{r}_{1}\right) \dot{\omega}_{2}+\omega_{2}^{2} \mathrm{mr} \mathrm{r}_{1}\left(\mathrm{r}_{0}+\frac{\mathrm{mr}}{\overline{\mathrm{M}}}\right) \phi_{1} \\
+\mathrm{m} \omega_{2} \mathrm{r}_{1} \Gamma \omega_{1}=-\mathrm{k} \dot{\phi}_{1}-\mathrm{K} \phi_{1}
\end{array}\right\}
$$

where the super-barred composite inertia terms now include the contributions of the main body, rotor, and the nutation damper.

The linearized variational equations can again be obtained by substituting Equations (4) into (13) and linearizing, assuming: $\delta \ll 1, \epsilon \ll 1, \psi \ll 1$. The following set of linearized equations result:

$$
\left.\begin{array}{l}
\dot{\delta}+\mathrm{C}_{1} \epsilon+\mathrm{C}_{5} \ddot{\phi}_{1}=0 \\
\dot{\epsilon}+\mathrm{C}_{2} \psi+\mathrm{C}_{3} \delta+\mathrm{C}_{6} \dot{\phi}_{1}=0 \\
\dot{\psi}+\mathrm{C}_{4} \epsilon+\mathrm{C}_{7} \ddot{\phi}_{1}+\mathrm{C}_{8} \phi_{1}=0 \\
\ddot{\phi}_{1}+\mathrm{C}_{9} \dot{\phi}_{1}+\mathrm{C}_{10} \phi_{1}+\mathrm{C}_{11} \dot{\delta}+\mathrm{C}_{12} \dot{\psi}+\mathrm{C}_{13} \epsilon=0
\end{array}\right\}
$$

where $\mathrm{C}_{1}-\mathrm{C}_{4}$ have already been defined, after Equation (5), and, in addition:

$$
\begin{aligned}
& \mathrm{C}_{5}=\mathrm{mr}_{1}\left(\mathrm{r}_{1}+\mathrm{r}_{0}\right) / \overline{\mathrm{B}} \\
& \mathrm{C}_{6}=-2 \mathrm{~m}(\Omega \cos \alpha) \mathrm{r}_{1} \Gamma / \overline{\mathrm{A}} \\
& \mathrm{C}_{7}=-\mathrm{mr}_{1} \Gamma / \overline{\mathrm{C}}
\end{aligned}
$$




$$
\begin{aligned}
& \mathrm{C}_{8}=\mathrm{mr}_{1}\left(\Omega^{2} \cos ^{2} a\right) \Gamma / \overline{\mathrm{C}} \\
& \mathrm{C}_{9}=\mathrm{k} / \mathrm{m} \mathrm{r}_{1}^{2} \\
& \mathrm{C}_{10}=\mathrm{K} / \mathrm{mr}_{1}^{2}+\Omega^{2} \cos ^{2} a\left(\mathrm{r}_{0} / \mathrm{r}_{1}+\mathrm{m} / \overline{\mathrm{M}}\right) \\
& \mathrm{C}_{11}=\left(\mathrm{r}_{0}+\mathrm{r}_{1}\right) / \mathrm{r}_{1} \\
& \mathrm{C}_{12}=-\Gamma / \mathrm{r}_{1} \\
& \mathrm{C}_{13}=\Gamma(\Omega \cos \alpha) / \mathrm{r}_{1}
\end{aligned}
$$

Again after some algebraic manipulation to eliminate the dependent degree of freedom, it can be shown that the system characteristic equation corresponding to the homogeneous linear system can be expressed as:

$$
f(\lambda)=a_{0} \lambda^{4}+a_{1} \lambda^{3}+a_{2} \lambda^{2}+a_{3} \lambda+a_{4}=0
$$

where

$$
\begin{aligned}
a_{0}= & 1-C_{5} C_{11}-C_{7} C_{12} \\
a_{1}= & C_{9} \\
a_{2}= & -\left(C_{2} C_{4}+C_{1} C_{3}\right)\left(1-C_{5} C_{11}-C_{7} C_{12}\right) \\
& +\left(C_{10}-C_{8} C_{12}\right) \\
& -\left(-C_{2} C_{7}-C_{3} C_{5}+C_{6}\right)\left(C_{13}-C_{1} C_{11}-C_{4} C_{12}\right) \\
a_{3}= & -C_{9}\left(C_{2} C_{4}+C_{1} C_{3}\right) \\
a_{4}= & C_{2}\left(-C_{4} C_{10}+C_{8} C_{13}\right)
\end{aligned}
$$

A necessary condition for stability is that all of the coefficients of the characteristic equation be non-zero and have the same sign. After substituting 
for the C's, it is found that

$$
a_{0}=1-\left(m\left(r_{0}+r_{1}\right)^{2} / \bar{B}\right)-m \Gamma^{2} / \bar{C}
$$

and that for the actual SAS-type system the last two terms are much less than one. Also,

$$
a_{1}=C_{9}=k / m r_{1}^{2} ; a_{1}>0
$$

if nutation damping is present.

$$
a_{3}=C_{9}\left(-C_{2} C_{4}-C_{1} C_{3}\right)
$$

if $\mathrm{C}_{9}>0$, the condition that: $-\mathrm{C}_{2} \mathrm{C}_{4}-\mathrm{C}_{1} \mathrm{C}_{3}>0$ is the same as inequality (10) or (11) already discussed.

For $a \ll 1$, but not zero, the necessary condition that $\mathrm{a}_{4}>0$ can be reduced, after some algebra, to:

$$
\frac{\mathrm{r}_{0}}{\mathrm{r}_{1}}+\frac{\mathrm{m}}{\overline{\mathrm{M}}}+\overline{\mathrm{K}}>\frac{\mathrm{m} \Gamma^{2}}{\overline{\mathrm{B}}-\overline{\mathrm{A}}+\mathrm{I}_{\mathrm{R}_{2}} \mathrm{~s} / \Omega}-\frac{\mathrm{m} \Gamma\left(\mathrm{r}_{0}+\mathrm{r}_{1}\right)(\overline{\mathrm{A}}-\overline{\mathrm{C}}) \alpha}{\overline{\mathrm{B}}\left[\overline{\mathrm{B}}-\overline{\mathrm{A}}+\mathrm{I}_{\mathrm{R}_{2}} \mathrm{~s} / \Omega\right]}
$$

When $\alpha=0, L_{d_{1}}=0$, and condition (16) is identical to the necessary and sufficient stability condition (18) of Reference 1.

The condition that $\mathrm{a}_{2}>0$ is automatically satisfied for the case when $a=0$, and $a_{0}>0$. For $a<1$, it can be shown that $a_{2}>0$ when $s \gg \gg$. For $a$ not restricted to be small the conditions that: $a_{2}>0, a_{3}>0, a_{4}>0$ are not as apparent, but could be developed and evaluated numerically.

Wheel energy dissipation has been previously modeled by assuming that the momentum wheel could have two degrees of flexural freedom relative to the 
main spacecraft hub. ${ }^{2}$ Figure 3 describes the momentum wheel deflection geometry for the assumed small deflections $\alpha_{z}$ and $\alpha_{x}$ showing the displacement of a differential mass $d m_{R}$ on the deflected wheel. If it is assumed that the rotor is attached at or very near to the system center of mass and that the wheel is homogeneous (such that $I_{R_{2}}=2 I_{R_{1}}$ ), together with the previous assumptions on the magnitudes of the $\omega_{i}, \dot{\phi}_{1}$ and $\phi_{1}$, the first order nonlinear equations of motion are obtained ${ }^{2}$ :

$$
\overline{\mathrm{B}} \dot{\omega}_{2}+\omega_{1} \omega_{3}(\overline{\mathrm{A}}-\overline{\mathrm{C}})+\mathrm{m} \mathrm{r}_{1}\left(\mathrm{r}_{1}+\mathrm{r}_{0}\right) \ddot{\phi}_{1}=\mathrm{L}_{2}
$$

$\overline{\mathrm{A}} \dot{\omega}_{1}+\omega_{2} \omega_{3}(\overline{\mathrm{C}}-\overline{\mathrm{B}})-\omega_{3} \mathrm{I}_{R_{2}} \mathrm{~s}-2 \mathrm{~m} \omega_{2} \mathrm{r}_{1} \Gamma \dot{\phi}_{1}$

$+\mathrm{I}_{\mathrm{R}_{1}}\left\{\left[\ddot{\alpha}_{z}+\left(\omega_{2}+\mathrm{s}\right)^{2} a_{z}\right] \sin s t-\left[\ddot{a}_{x}+\left(\omega_{2}+s\right)^{2} a_{x}\right] \cos s t\right\}=L_{1}$

$\overline{\mathrm{C}} \dot{\omega}_{3}+\omega_{1} \omega_{2}(\overline{\mathrm{B}}-\overline{\mathrm{A}})+\omega_{1} \mathrm{I}_{\mathrm{R}_{2}} \mathrm{~s}-\mathrm{m} \mathrm{r}_{1} \Gamma \ddot{\phi}_{1}+\mathrm{m} \omega_{2}^{2} \mathrm{r}_{1} \Gamma \phi_{1}$

$+I_{R_{1}}\left\{\left[\ddot{\alpha}_{z}+\left(\omega_{2}+s\right)^{2} a_{z}\right] \cos s t+\left[\ddot{a}_{x}+\left(\omega_{2}+s\right)^{2} a_{x}\right] \sin s t\right\}=L_{3}$

$m r_{1}^{2}\left(1-\frac{m}{\bar{M}}\right) \ddot{\phi}_{1}-m r_{1} \Gamma \dot{\omega}_{3}+m r_{1}\left(r_{0}+r_{1}\right) \dot{\omega}_{2}+\omega_{2}^{2} m r_{1}\left(r_{0}+\frac{m r_{1}}{\bar{M}}\right) \phi_{1}$

$$
+\mathrm{m} \omega_{2} \mathrm{r}_{1} \Gamma \omega_{1}=-\mathrm{k} \dot{\phi}_{1}-\mathrm{K} \phi_{1}
$$




$$
\begin{aligned}
& \mathrm{I}_{\mathrm{R}_{1}} \ddot{\alpha}_{z}+\mathrm{k}_{\mathrm{R}} \dot{\alpha}_{\mathrm{z}}+\left[\mathrm{K}_{\mathrm{R}}+\mathrm{I}_{\mathrm{R}_{1}}\left(\omega_{2}+\mathrm{s}\right)^{2}\right] a_{z} \\
& \quad+\mathrm{I}_{\mathrm{R}_{1}}\left\{\left[\dot{\omega}_{1}-\left(\omega_{2}+2 \mathrm{~s}\right) \omega_{3}\right] \sin \mathrm{st}+\left[\dot{\omega}_{3}+\left(\omega_{2}+2 \mathrm{~s}\right) \omega_{1}\right] \cos \mathrm{st}\right\}=0 \\
& \mathrm{I}_{\mathrm{R}_{1}} \ddot{\alpha}_{\mathrm{x}}+\mathrm{k}_{\mathrm{R}} \dot{a}_{\mathrm{x}}+\left[\mathrm{K}_{\mathrm{R}}+\mathrm{I}_{\mathrm{R}_{1}}\left(\omega_{2}+\mathrm{s}\right)^{2}\right] a_{\mathrm{x}} \\
& \quad+\mathrm{I}_{\mathrm{R}_{1}}\left\{\left[\ddot{\omega}_{3}+\left(\omega_{2}+2 \mathrm{~s}\right) \omega_{1}\right] \sin \mathrm{st}-\left[\dot{\omega}_{1}-\left(\omega_{2}+2 \mathrm{~s}\right) \omega_{3}\right] \cos \mathrm{st}\right\}=0
\end{aligned}
$$

If it is assumed that $\mathrm{L}_{2}=\mathrm{L}_{3}=0$ and that $\mathrm{L}_{1}=\mathrm{L}_{\mathrm{d}_{1}}$, Equations (17)-(22) may be linearized about the equilibrium motion:

$$
\begin{aligned}
& \omega_{2}=\Omega \cos \alpha+\delta, \omega_{1}=\epsilon, \omega_{3}=\Omega \sin a+\psi \\
& \phi_{1}=a_{z}=a_{\mathbf{x}}=0
\end{aligned}
$$

to yield in terms of the previously defined constants:

$$
\begin{gathered}
\dot{\delta}+\mathrm{C}_{1} \epsilon+\mathrm{C}_{5} \ddot{\phi}_{1}=0 \\
\dot{\epsilon}+\mathrm{C}_{2} \psi+\mathrm{C}_{3} \delta+\mathrm{C}_{6} \dot{\phi}_{1}+\left(\mathrm{I}_{\mathrm{R}_{1}} / \overline{\mathrm{A}}\right)\left\{\operatorname{sinst}\left[\ddot{a}_{z}+(\Omega \cos a+\mathrm{s})^{2} a_{z}\right]\right. \\
\left.-\operatorname{cosst}\left[\ddot{\alpha}_{\mathrm{x}}+(\Omega \cos \alpha+\mathrm{s})^{2} a_{\mathrm{x}}\right]\right\}=0 \\
\dot{\psi}+\mathrm{C}_{4} \epsilon+\mathrm{C}_{7} \ddot{\phi}_{1}+\mathrm{C}_{8} \phi_{1}+\left(\mathrm{I}_{\mathrm{R}_{1}} / \overline{\mathrm{C}}\right)\left\{\cos \mathrm{st}\left[\ddot{\alpha}_{z}+(\Omega \cos a+\mathrm{s})^{2} a_{2}\right]\right. \\
\left.+\operatorname{sinst}\left[\ddot{\alpha}_{\mathrm{x}}+(\Omega \cos a+\mathrm{s})^{2} a_{\mathrm{x}}\right]\right\}=0
\end{gathered}
$$




$$
\begin{aligned}
& \ddot{\phi}_{1}+\mathrm{C}_{9} \dot{\phi}_{1}+\mathrm{C}_{10} \phi_{1}+\mathrm{C}_{11} \dot{\delta}+\mathrm{C}_{12} \dot{\psi}+\mathrm{C}_{13} \epsilon=0 \\
& \ddot{a}_{z}+\left(\mathrm{k}_{\mathrm{R}} / \mathrm{I}_{\mathrm{R}_{1}}\right) \dot{\alpha}_{z}+\left[\mathrm{K}_{\mathrm{R}} / \mathrm{I}_{\mathrm{R}_{1}}+(\Omega \cos \alpha+\mathrm{s})^{2}\right] a_{z} \\
& +[\dot{\epsilon}-(\Omega \cos \alpha+2 s) \psi+(\Omega \sin \alpha) \delta] \sin s t \\
& +[\ddot{\psi}+(\Omega \cos \alpha+2 s) \epsilon] \cos s t=(\sin s t) \Omega \sin \alpha(\Omega \cos \alpha+2 s) \\
& \ddot{a}_{\mathrm{x}}+\left(\mathrm{k}_{\mathrm{R}} / \mathrm{I}_{\mathrm{R}_{1}}\right) \dot{\alpha}_{\mathrm{x}}+\left[\mathrm{K}_{\mathrm{R}_{1}} / \mathrm{I}_{\mathrm{R}_{1}}+(\Omega \cos \alpha+\mathrm{s})^{2}\right] a_{\mathrm{x}} \\
& +[\dot{\psi}+(\Omega \cos \alpha+2 s) \epsilon] \sin s t-[\dot{\epsilon}-(\Omega \cos \alpha+2 s) \psi+(\Omega \sin \alpha) \delta] \cos s t \\
& =-(\cos s t) \Omega \sin \alpha(\Omega \cos \alpha+2 s)
\end{aligned}
$$

Equations (23)-(28) represent a ninth order non-homogeneous set of nonautonomous differential equations. The stability of the homogeneous system could be analyzed using Floquet theory similar to the treatment of Mingori. ${ }^{6}$ The application of Floquet theory for this problem would necessitate the use of a digital computer to study the stability point by point by varying different satellite and rotor inertia, spin, and damping system parameters in a systematic manner.

Instead of the Floquet analysis the Lyapunov Reducibility Theorem may be applied to reduce the linear system of differential equations with periodic coefficients to a kinematically similar system of autonomous differential equations ${ }^{7}$. This method has been recently employed by Guha ${ }^{8}$ in studying the attitude stability of dissipative dual-spin spacecraft. 
The homogeneous system of linear nonautonomous differential equations can be expressed in standard state vector form:

$$
\dot{\mathbf{x}}=\mathrm{A}(\mathrm{t}) \mathbf{x}
$$

where, for this system, A (t) represents a $12 \times 12$ matrix. According to the Lyapunov Reducibility Theorem ${ }^{7,8}$ there is a bounded nonsingular transformation matrix $S(t)$ with a bounded inverse $S^{-1}(t)$ such that a matrix, $D$, defined by the equation:

$$
D=S^{-1} A S-S^{-1} \dot{S}
$$

is a constant matrix. We can then relate,

$$
\mathbf{x}=\mathrm{S}(\mathrm{t}) \mathrm{y}
$$

and obtain the system,

$$
\dot{\mathrm{y}}=\mathrm{Dy}
$$

The system of autonomous equations (32), is said to be kinematically similar to the original system, (29) and has similar stability properties since the characteristic roots of the matrix $\mathrm{D}$ are the characteristic exponents of the original equation, (29). According to a theorem of Lyapunov, every equation with periodic coefficients is reducible, and the major problem is to determine the bounded transformation, $\mathrm{S}(\mathrm{t})$.

Guha has shown ${ }^{8}$ in applying this technique to the system considered in Reference 2, that a suitable transformation of the form of Equation (31) can be expressed: 


$$
\left[\begin{array}{l}
\omega_{1} \\
\omega_{3} \\
\phi_{1} \\
\alpha_{z} \\
a_{x}
\end{array}\right]=\left[\begin{array}{ccccc}
1 & 0 & 0 & 0 & 0 \\
0 & 1 & 0 & 0 & 0 \\
0 & 0 & 1 & 0 & 0 \\
0 & 0 & 0 & \text { cos st } & \sin s t \\
0 & 0 & 0 & \text { sin st } & -\cos s t
\end{array}\right]\left[\begin{array}{l}
\omega_{1} \\
\omega_{3} \\
\phi_{1} \\
\beta_{1} \\
\beta_{2}
\end{array}\right]
$$

It can be verified that this same type of nonsingular bounded transformation can be applied to the present system, Equations (23)-(28). The following autonomous, and kinematically similar system in the variational coordinates $\delta, \epsilon, \psi, \phi_{1}, \beta_{1}$, and $\beta_{2}$, results:

$$
\begin{gathered}
\dot{\delta}+\mathrm{C}_{1} \epsilon+\mathrm{C}_{5} \ddot{\phi}_{1}=0 \\
\dot{\epsilon}+\mathrm{C}_{2} \psi+\mathrm{C}_{3} \delta+\mathrm{C}_{6} \dot{\phi}_{1}+\mathrm{C}_{14} \dot{\beta}_{1}+\mathrm{C}_{15} \ddot{\beta}_{2}+\mathrm{C}_{16} \beta_{2}=0 \\
\dot{\psi}+\mathrm{C}_{4} \epsilon+\mathrm{C}_{7} \ddot{\phi}_{1}+\mathrm{C}_{8} \phi_{1}+\mathrm{C}_{17} \ddot{\beta}_{1}+\mathrm{C}_{18} \beta_{1}+\mathrm{C}_{19} \dot{\beta}_{2}=0 \\
\ddot{\phi}_{1}+\mathrm{C}_{9} \dot{\phi}_{1}+\mathrm{C}_{10} \phi_{1}+\mathrm{C}_{11} \dot{\delta}+\mathrm{C}_{12} \dot{\psi}+\mathrm{C}_{13} \epsilon=0 \\
\ddot{\beta}_{1}+\mathrm{C}_{20} \dot{\beta}_{1}+\mathrm{C}_{21} \beta_{1}+\mathrm{C}_{22} \dot{\beta}_{2}+\mathrm{C}_{23} \beta_{2}+\dot{\psi}+\mathrm{C}_{24} \epsilon=0 \\
\ddot{\beta}_{2}+\mathrm{C}_{20} \dot{\beta}_{2}+\mathrm{C}_{21} \beta_{2}-\mathrm{C}_{22} \dot{\beta}_{1}-\mathrm{C}_{23} \beta_{1}+\dot{\epsilon}-\mathrm{C}_{24} \psi+\mathrm{C}_{25} \delta=\Omega \sin \alpha(\Omega \cos \alpha+2 \mathrm{~s})(39)
\end{gathered}
$$

where $C_{1}-C_{13}$ have been defined previously and, in addition,

$$
\begin{aligned}
& \mathrm{C}_{14}=-2 \mathrm{I}_{\mathrm{R}_{1}} \mathrm{~s} / \overline{\mathrm{A}} \\
& \mathrm{C}_{15}=\mathrm{I}_{\mathrm{R}_{1}} / \overline{\mathrm{A}}
\end{aligned}
$$




$$
\begin{aligned}
& \mathrm{C}_{16}=\mathrm{I}_{\mathrm{R}_{1}} \Omega \cos a(\Omega \cos a+2 \mathrm{~s}) / \overline{\mathrm{A}} \\
& \mathrm{C}_{17}=\mathrm{I}_{\mathrm{R}_{1}} / \overline{\mathrm{C}} \\
& \mathrm{C}_{18}=\mathrm{I}_{\mathrm{R}_{1}} \Omega \cos a(\Omega \cos a+2 \mathrm{~s}) / \overline{\mathrm{C}} \\
& \mathrm{C}_{19}=2 \mathrm{I}_{\mathrm{R}_{1}} \mathrm{~s} / \overline{\mathrm{C}} \\
& \mathrm{C}_{20}=\mathrm{k}_{\mathrm{R}} / \mathrm{I}_{\mathrm{R}_{1}} \\
& \mathrm{C}_{21}=\left(\mathrm{K}_{\mathrm{R}} / \mathrm{I}_{\mathrm{R}_{1}}\right)+\Omega \cos a(\Omega+2 \mathrm{~s}) \\
& \mathrm{C}_{22}=2 \mathrm{~s} \\
& \mathrm{C}_{23}=\mathrm{k}_{\mathrm{R}} \mathrm{s} / \mathrm{I}_{\mathrm{R}_{1}} \\
& \mathrm{C}_{24}=\Omega \cos a+2 \mathrm{~s} \\
& \mathrm{C}_{25}=\Omega \mathrm{sin} a
\end{aligned}
$$

Equation (38) is obtained by multiplying (27) by cos st and (28) by sin st and adding; Equation (39) is similarly obtained by multiplying (27) by sin st and (28) by -cos st and adding.

The stability analysis of this system is a standard exercise in linear systems theory. The stability of the zero solution of the corresponding homogeneous system may be analyzed by Routh's method. The characteristic polynomial may be evaluated, and the system stability dependent on the roots of the characteristic equation all having negative real parts. 
For the non-homogeneous system, the steady state solution may be obtained by assuming:

$$
\delta_{\mathrm{ss}}=\mathrm{K}_{1}, \epsilon_{\mathrm{ss}}=\mathrm{K}_{2}, \psi_{\mathrm{ss}}=\mathrm{K}_{3}, \phi_{1_{\mathrm{ss}}}=\mathrm{K}_{4}, \beta_{1_{\mathrm{ss}}}=\mathrm{K}_{5}
$$

and $\beta_{2 s}=K_{6}$ and substituting back into Equations $(34)-(39)$. It can be seen that:

$$
\begin{aligned}
& \delta_{s s}=C_{24} C_{25} /\left(C_{25}+C_{24} C_{3} / C_{2}\right), \\
& \psi_{s s}=-\frac{C_{3}}{C_{2}} \delta_{s s}
\end{aligned}
$$

and $\epsilon_{\mathrm{ss}}=\phi_{1_{\mathrm{ss}}}=\beta_{1_{\mathrm{ss}}}=\beta_{2_{\mathrm{s}}}=0$. An examination of the constants involved in $\delta_{s s}$ and $\psi_{s s}$ indicates that the steady state motion of the kinematically similar system is bounded.

\section{NUMERICAL RESULTS}

The characteristic equation was developed for the linear autonomous homogeneous system corresponding to Equations (34)-(39). Equilibrium values of $\alpha_{0}$ corresponding to the reportedly observed nutation angles of 0.1 degree and 0.2 degree were calculated as follows:

$$
\tan \gamma_{0}=\frac{\mathrm{C} \Omega \sin \alpha_{0}}{\mathrm{I}_{\mathrm{R}_{2}} \mathrm{~s}+\mathrm{B} \Omega \cos \alpha_{0}}
$$

where the momentum components are approximated by:

$$
\begin{aligned}
& \mathrm{H}_{2} \approx \mathrm{I}_{\mathrm{R}_{2}} \mathrm{~s}+\mathrm{B}(\Omega \cos a) \\
& \mathrm{H}_{1} \approx 0 \\
& \mathrm{H}_{3} \approx \mathrm{C}(\Omega \sin a)
\end{aligned}
$$


since $\mathrm{A} \approx \overline{\mathrm{A}}, \mathrm{B} \approx \overline{\mathrm{B}}$, and $\mathrm{C} \approx \overline{\mathrm{C}}$.

Two cases of interest were considered, where, first one pair of SAS-A solar paddles was assumed to be loosely attached to the hub with the other pair rigidly connected, and secondly, vice versa. The moments of inertia for the two different cases are:

\section{Case I}

$$
\begin{aligned}
& A=28.11929 \mathrm{~kg}-\mathrm{m}^{2} \\
& B=17.747422 \mathrm{~kg}-\mathrm{m}^{2} \\
& \mathrm{C}=15.32054 \mathrm{~kg}-\mathrm{m}^{2}
\end{aligned}
$$

\section{Case II}

$$
\begin{aligned}
& A=15.18496 \mathrm{~kg}-\mathrm{m}^{2} \\
& B=17.74742 \mathrm{kg-m}{ }^{2} \\
& C=28.634496 \mathrm{~kg}-\mathrm{m}^{2}
\end{aligned}
$$

It is seen that from Equation (40), for Case I, $\alpha_{0}=1^{\circ} 55^{\prime}$ and for Case II, $\alpha_{0}=$ $1^{\circ} 1^{\prime}$, for $\gamma_{0}=0.1$ degree; also for $\gamma_{0}=0.2$ degree, $\alpha_{0}=2^{\circ} 6^{\prime}$, and $3^{\circ} 49^{\prime}$ for CaseII and I respectively.

The roots of the characteristic equation were calculated for the two cases using the following nominal SAS-A orbital parameters:

$$
\begin{array}{rlrl}
\Gamma & =0.453 \mathrm{~m} . & \mathrm{k} & =5.7 \times 10^{-5} \mathrm{nt}-\mathrm{m}-\mathrm{sec} / \mathrm{rad} \\
\mathrm{r}_{0} & =0.0189 \mathrm{~m} . & \mathrm{K} & =5.8 \times 10^{-5} \mathrm{nt}-\mathrm{m} / \mathrm{rad} . \\
\mathrm{r}_{1} & =0.1735 \mathrm{~m} . & * \mathrm{k}_{\mathrm{R}}=6.779 \times 10^{-3} \mathrm{nt}-\mathrm{m}-\mathrm{sec} / \mathrm{rad} \\
\mathrm{m} & =0.2451 \mathrm{~kg} . & * \mathrm{~K}_{\mathrm{R}}=71.61074 \mathrm{nt}-\mathrm{m} / \mathrm{rad} . \\
\mathrm{I}_{\mathrm{R}_{2}} & =0.011525 \mathrm{~kg}-\mathrm{m}^{2} & \mathrm{M}=114 \mathrm{~kg} . \\
\mathrm{I}_{\mathrm{R}_{1}} & =.0 .0050799 \mathrm{~kg}-\mathrm{m}^{2}=\mathrm{I}_{\mathrm{R}_{3}} & & \\
\mathrm{~S} & =209.43 \mathrm{rad} / \mathrm{sec} . & & \\
\Omega & =0.008727 \mathrm{rad} / \mathrm{sec} . & &
\end{array}
$$

\footnotetext{
*Determined experimentally at The Johns Hopkins University, Applied Physics Laboratory (see Ref. 2).
} 
For this system, after elimination of the dependence between coordinates, similar to Equations (6) and (7), an eighth order characteristic equation results. The roots were obtained using digital computations with a polynomial root-finder subroutine on the XDS-9300 computer. The results are summarized as follows for $\gamma_{0}=0.1$ degree (for $\gamma_{0}=0.2$ degree, changes are observed only in the third or fourth significant figure):

Real Part

Case I

$-.37874 \times 10^{-2}$

$-.8058 \times 10^{-4}$

$-.58874$

$-.58843$

\section{Case II}

$-.38139 \times 10^{-2}$

$-.4834 \times 10^{-4}$

$-.58875$

$-.58844$
Imaginary Part - $(\mathrm{Rad} / \mathrm{sec})$

$$
\begin{aligned}
& \pm .88380 \times 10^{-1} \\
& \pm .11455 \\
& \pm .27962 \times 10^{2} \\
& \pm .44670 \times 10^{3}
\end{aligned}
$$

$$
\begin{aligned}
& \pm .88468 \times 10^{-1} \\
& \pm .11374 \\
& \pm .27963 \times 10^{2} \\
& \pm .4467 \times 10^{3}
\end{aligned}
$$

It is seen that all of the roots for both cases contain only negative real parts. The higher frequency modes are essentially the same for the two cases. The time constant for the least damped mode is about 12,400 seconds ( $3.44 \mathrm{hrs}$.) for the first case and 20,700 seconds (5.75 hrs.) for the second case. This represents a considerable degradation in the performance of the nutation damping system about the equilibrium position $\gamma_{0}=0$ previously considered. ${ }^{1,2}$ 
The highest frequency mode for the kinematically similar system has the frequency of $446.7 \mathrm{rad} / \mathrm{sec}$, approximately 2.14 times the wheel relative angular velocity of $209.4 \mathrm{rad} / \mathrm{sec}$. It should be noted that in the original system the wheel deflection equations, Equations (27) and (28), have forcing terms on the right hand side at frequency $\mathrm{s}=209.4 \mathrm{rad} / \mathrm{sec}$. The possibility of forced resonance in the original system might exist, especially as the highest frequency of the kinematically similar system approaches $2 \mathrm{~s}$. This eventuality could be examined more closely by numerical integration of the system equations.

It has been noted in the SAS-A post launch performance, that in addition to the somewhat larger nutation angles experienced than predicted by pre-launch analyses, a noticeable degradation of the nutation damping time constant occurs. Time constants as large as three hours or more have been observed. ${ }^{9}$ While the present analysis does not claim an exact correlation with the anomalous observed behavior, it does suggest a possible mechanism for future problems, especially in connection with high pointing accuracy dual-spin missions.

It has also been demonstrated by computer simulations that external torques having amplitudes of about 4000 dyne-cm would be necessary to produce nutational motion of the SAS-A satellite with amplitudes of 0.1 degree. ${ }^{9}$ Torques of this magnitude would be difficult to justify using even conservative aerodynamic density values at the SAS-A orbital altitude. The magnitude of the reaction torque, $L_{d_{1}}$, as given by Equation (3) was calculated for the two cases previously considered; for Case I, $L_{d_{1}}=-7090$ dyne $-\mathrm{cm}$, whereas for Case II, $L_{d_{1}}=-3620$ dyne-cm. There is a strong suggestion, therefore, that the solar panels and not the atmosphere are providing the higher amplitude torques associated with the anomalous nutational motion. 


\section{CONCLUSIONS - CLOSING COMMENTS}

Based on the present stability analysis and numerical results the following concluding comments can be made:

1. The motion of a dual-spin satellite system, under the influence of applied reaction torques, about an axis of rotation slightly displaced from the nominal spin axis remains bounded. For small initial perturbations, the system, however, is not asymptotically stable about the original equilibrium state.

2. For the case of energy dissipation only on the main body it is seen that the necessary Routh stability criteria for this motion are satisfied; for the general case of damping on both the main spacecraft and the wheel, stability is indicated by a numerical examination of the roots of the system characteristic equation of the kinematically similar system.

3. The Lyapunov Reducibility Theorem can be used in studying the stability of the original nonautonomous system for the case of energy dissipation on both parts of the dual-spin system.

4. A possible mechanism for the somewhat anomalous nutational motion and large nutational time constants observed in the post-launch SAS-A flight data is suggested. For this system it is assumed that the applied reaction torques would be provided by either pair of non-rigidly attached solar paddles. 


\section{REFERENCES}

1. Bainum, P. M., Fuechsel, P. G., and Mackison, D. L., "Motion and Stability of a Dual-Spin Satellite with Nutation Damping," Journal of Spacecraft and Rockets, Vol. 7, No. 6, Jinne 1970, pp. 690-696; also as TG-1072, June 1969, Applied Physics Laboratory, The Johns Hopkins University, Silver Spring, Md.

2. Bainum, P. M., "Stability of SAS-A Dual-Spin Spacecraft with Energy Dissipation on the Momentum Wheel," NASA Goddard Space Flight Center, Greenbelt, Maryland, Report No. X-732-70-344, September 1970; also Bainum, P. M., Fuechsel, P. G., and Fedor, J. V., "Stability of a Dual-Spin Spacecraft with Energy Dissipation in a Flexible Momentum Wheel," AAS/ALAA Astrodynamics Specialists Conference 1971, Ft. Lauderdale, Fla., August 17-19, 1971, AAS Paper No. 71-347.

3. Fuechsel, P. G., Bainum, P. M., and Grunberger, P. J., "The Attitude Motion of a Nutationally Damped Dual-Spin Spacecraft in the Presence of Near-Earth Environment," AIAA Aerospace Sciences Meeting, New York, N.Y., January 25-27, 1971, Paper No. 71-90; to appear Journal of Spacecraft and Rockets.

4. Synge, John L. and Griffith, Byron A., Principles of Mechanics, Second Edition, McGraw Hill Book Company, Inc., 1949, pp. 352-353.

5. Garber, T. B., "The Influence of Constant Disturbing Torques on the Motion of Gravity-Gradient Stabilized Satellites," AIAA Journal, Vol. 1, April 1963. 
6. Mingori, D. L., "Effects of Energy Dissipation on the Attitude Stability of Dual-Spin Satellites," ALAA Journal, Vol. 7, No. 1, January 1969, pp. 20-27.

7. Hahn, W., Stability of Motion, Springer-Verlag, Berlin, Heidelberg, New York, 1967, pp. 120-122; 296-304.

8. Guha, A. K., "Attitude Stability of Dissipative Dual-Spin Spacecrafts," submitted for publication AIAA Journal, 1971.

9. Private communication with P. G. Fuechsel of the Johns Hopkins University, Applied Physics Laboratory, August 1971. 


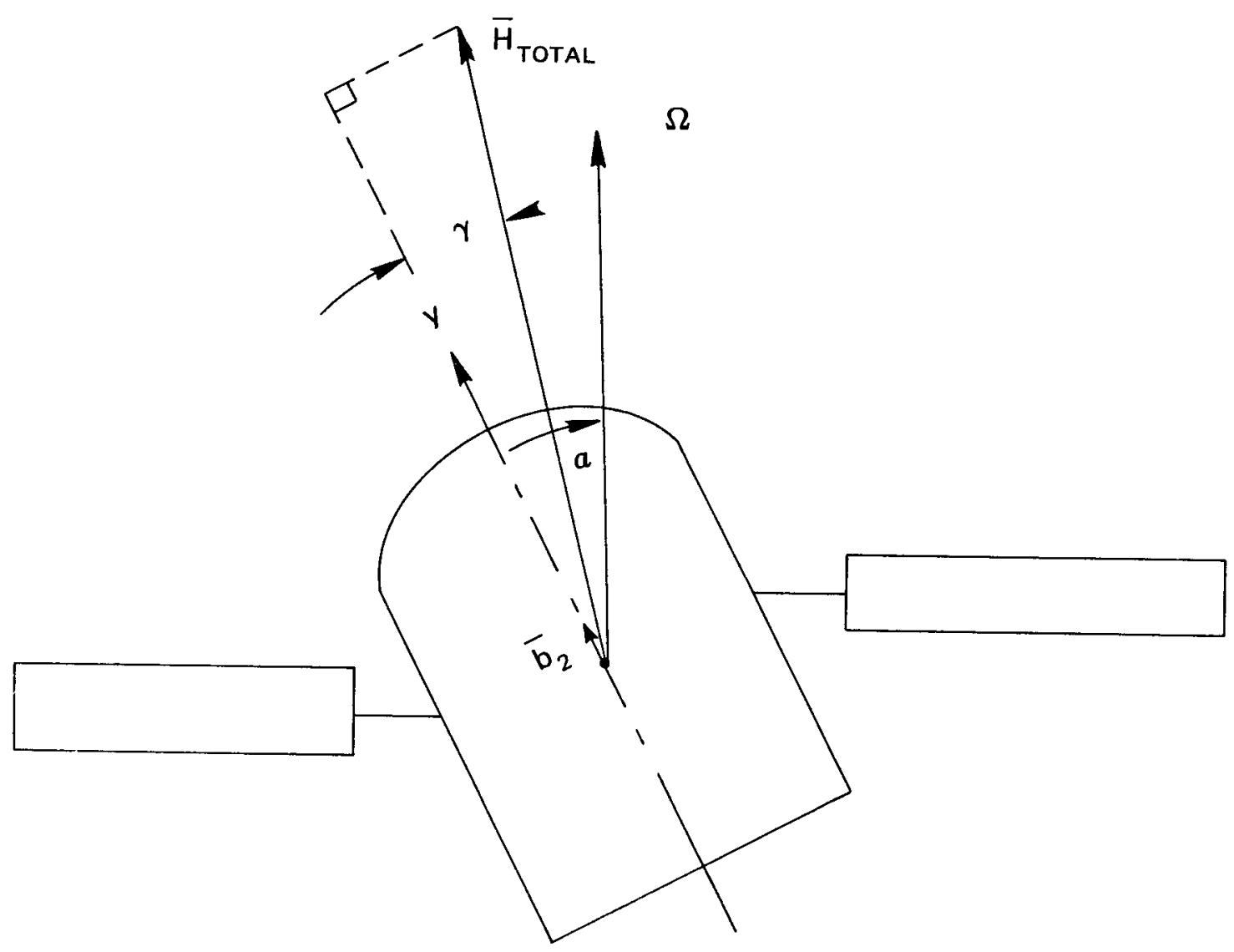

Figure 1. Possible SAS-A In-Orbit Equilibrium 


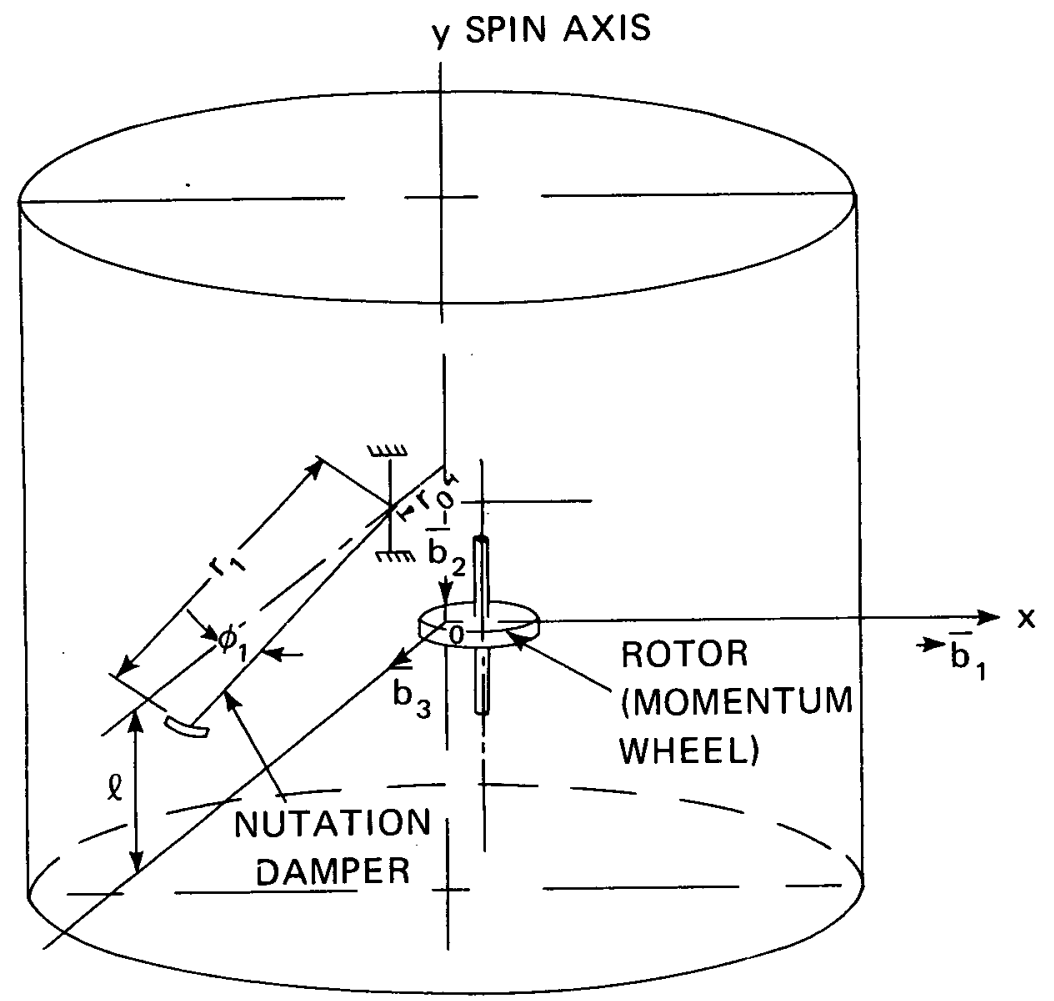

Figure 2. Elements of SAS-A Attitude Control System 


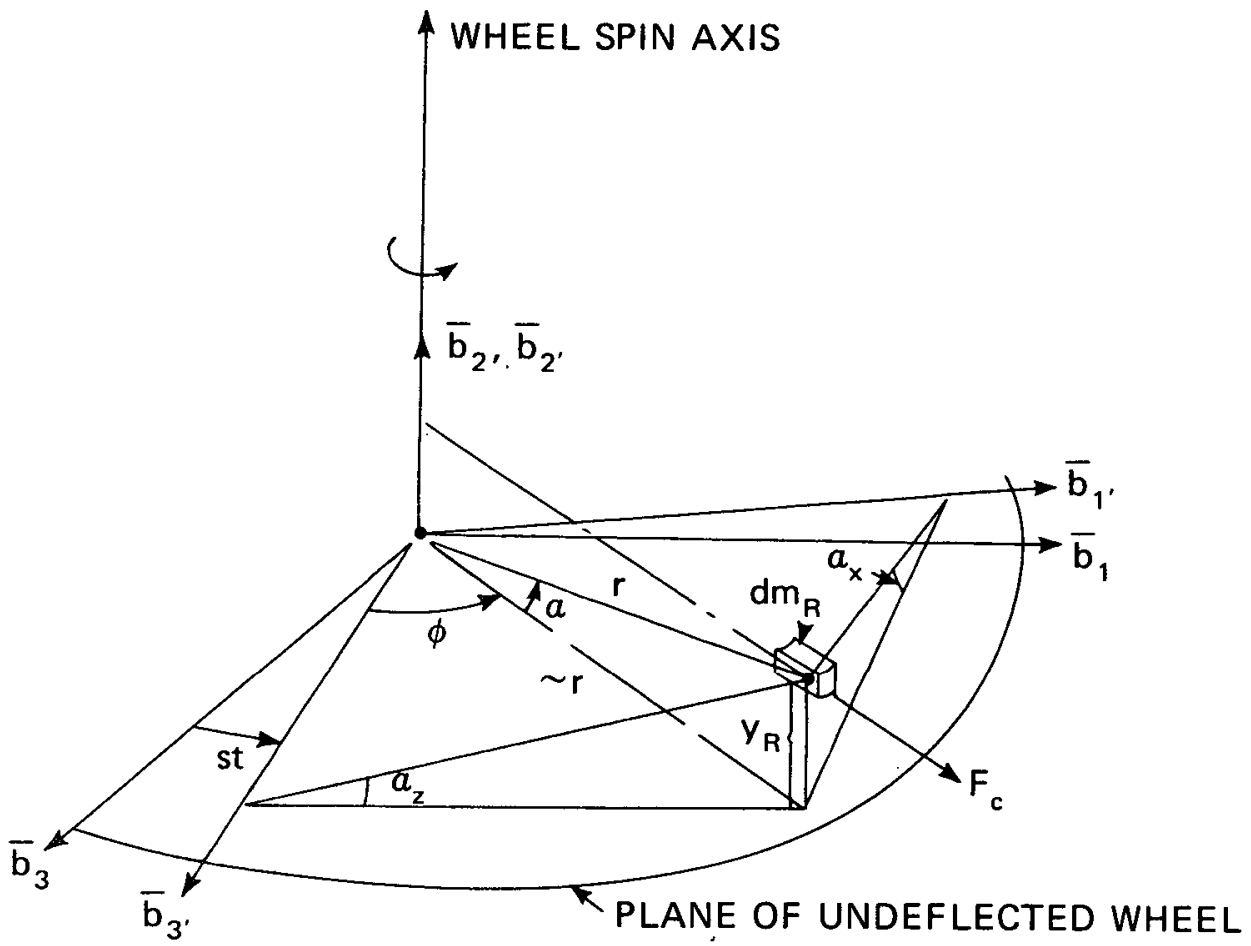

Figure 3. Momentum Wheel Deflection Geometry 